\title{
ANÁLISIS DE INSTRUMENTOS DE MEDICIÓN DEL PENSAMIENTO CRÍTICO
}

\author{
ANALYSIS OF CRITICAL THINKING MEASURING INSTRUMENTS \\ Carlos J. Ossa-Cornejo* \\ Maritza R. Palma-Luengo* \\ Nelly G. Lagos-San Martín* \\ Ingrid M. Quintana-Abello** \\ Claudio H. Díaz-Larenas*** \\ *Departamento de Ciencias de la Educación, Universidad del Bio-Bio. Chile \\ **Departamento de Ciencias Sociales, Universidad del Bio-Bio. Chile \\ ***Unidad de Investigación y Desarrollo Docente, Universidad de Concepción. Chile
}

\begin{abstract}
Resumen: El pensamiento crítico es una competencia relevante hoy en día en la formación universitaria, con escaso consenso conceptual y metodológico en sus instrumentos de medición. En este artículo se ofrece una revisión acerca de los instrumentos que se han utilizado para medir el pensamiento crítico, con la finalidad de generar una discusión que permita una mejor comprensión y valoración de los aspectos que componen esta habilidad. La metodología utilizada consiste en una revisión sistemática de estudios en bases de datos, seleccionando 31 estudios de 97 encontrados, y analizando en profundidad ocho de ellos; se utilizó además, análisis de contenido temático para las definiciones y características de instrumentos. Los resultados señalan la existencia de divergencias a la hora de definir y evaluar el pensamiento crítico, con variedad de instrumentos y escaso consenso en los componentes medidos. Se discute la necesidad de lograr un modelo de Pensamiento Crítico integrado que considere habilidades cognitivas, metacognitivas y disposicionales.
\end{abstract}

Palabras Clave: pensamiento crítico; evaluación; cognición; instrumentos; análisis de contenido

\begin{abstract}
Critical thinking is a relevant ability nowadays in higher education, but there is a lack of consensus on its definitions and assessment instruments. This article offers a conceptual and methodological review about the instruments used to measure critical thinking, in order to generate a discussion that allows a better understanding and appreciation of the aspects that are considered in this skill. The methodology consisted of a systematic review of studies in databases, selecting 31 studies out of 97 founded, and 8 of them for deep analysis; atopic content analysis in definitions and test characteristics is also used. Results show the existence of many differences in definitions and assessments of critical thinking, with a variety of tests and little consensus on the measured components. The need of an integrative model of Critical Thinking, considering cognitive, metacognitive and dispositional skills is discussed.
\end{abstract}

Key Words: critical thinking; assessment; cognition; test; content analysis

Este estudio se ha realizado dentro del proyecto de investigación DIUBB 152023 3/IDU con el apoyo de la Dirección de Investigación de la Universidad del Bío-Bío. Chile

\section{Introducción}

El pensamiento crítico es valorado como una forma superior de razonamiento, una competencia transversal a los sistemas educativos (Almeida, \& Rodríguez, 2011) y un recurso cognitivo esencial (Davies, 2013; Phan, 2010) en la formación profe- sional puesto que es una herramienta fundamental frente a la cantidad de información y la multiplicidad de situaciones a enfrentar diariamente en la actualidad, que exigen nuevas funciones cognitivas, particularmente poder combinar conocimiento, experiencia y habilidades intelectuales para un desempeño eficiente. 
No obstante, dicho concepto es de difícil definición, puesto que se puede entender desde diversas perspectivas (Bailin, Case, Coombs, \& Daniels, 1999; Facione, 1990; Hager \& Kaye, 1992; Paul \& Elder 2003). Es así como tradicionalmente se le ha descrito tanto como un proceso de pensamiento lógico y científico, como una respuesta de desaprobación y contestataria o un mecanismo que permite reflexionar y filosofar (López, 2012).

Se ha definido al pensamiento crítico desde diferentes disciplinas (Facione, 2007; Madariaga \& Schaferchnit, 2013), tales como la filosofía, la psicología, la sociología y la educación como un tipo de pensamiento elaborado, es decir, como un proceso cognitivo que implica evaluación y reflexión (Glaser, en Hager \& Kaye, 1992). Es una habilidad que permite la construcción de un conocimiento nuevo, y la utilización estratégica del mismo en la solución de problemas presentes en la vida cotidiana (Black, 2012; Halpern, 2006), por lo que ha sido una herramienta relevante en la formación profesional de carreras del área de la salud, de la ingeniería, psicología, educación entre otras.

Del mismo modo, se han utilizado diferentes instrumentos para evaluarlo, tanto de forma genérica como en los componentes que lo sustentan (Butler, 2012; Madariaga \& Schaferchnit, 2013), encontrándose una gran variedad de maneras de implementar programas para su promoción.

\section{Pensamiento crítico}

Este constructo es definido como un tipo de proceso cognitivo complejo, integrado por subprocesos interrelacionados que permiten evaluar, procesar analítica y reflexivamente, enjuiciar y aceptar o rechazar, información producida en contextos sociales o en trabajos científicos (Tung \& Chang, 2009; Yang, 2012). Otra revisión del concepto, indica que el pensamiento crítico corresponde a un conjunto de habilidades intelectuales, aptitudes y disposiciones caracterizadas por el dominio profundo del contenido y del aprendizaje, que desarrolla la apreciación por la razón y la evidencia (Paul \& Elder, 2003).

Bajo el enfoque del proyecto DELPHI en Estados Unidos, donde un conjunto de expertos norteamericanos en pensamiento crítico, se reunieron para definir y caracterizar el constructo (Facione, 1990), éste puede definirse como un pensamiento elaborado o juicio que depende de la propia persona que busca un objetivo, dando como resultado habilidades de interpretación, análisis, evaluación e inferencia; también permitiría la explicación de situaciones en base a evidencia conceptual, metodológica, criteriológica o contextual, sobre las cuales se basa ese juicio (Facione, 1990; 2007). De esta manera se identifican habilidades intelectuales como Mente abierta; Análisis; Madurez cognitiva; Búsqueda de la verdad; Sistematicidad; Curiosidad; y, Confianza en sí mismo.

Se plantea asimismo, que es un modo de pensar en el cual el sujeto mejora la calidad de dicho proceso al apoderarse de las estructuras inherentes al acto de pensamiento y al someterlas a estándares intelectuales (Paul \& Elder, 2003). Se considera además como una habilidad de pensamiento que permite evaluar el mérito, la precisión, y/o autenticidad de la información que se está aprendiendo o elaborando, por lo que resulta una habilidad importante para el desarrollo de profesionales científicos (Antequera, 2011; Cassany, 2005).

Se puede establecer la existencia de un conjunto de elementos básicos de naturaleza cognitiva que integran el pensamiento crítico, destacando entre ellas la reflexión, la evaluación de la información, el análisis de opciones y la creatividad (Sánchez, 2012; Tiwari, Lai, So, \& Yuen, 2006). Estos componentes básicos en la descripción del pensamiento crítico, permitirían alcanzar funciones cognitivas complejas como el razonamiento, la solución de problemas y la toma de decisiones (Saadé, Morin, \& Thomas, 2012; Saiz \& Rivas, 2008).

Entre estos componentes, el primer elemento que caracterizaría al pensamiento crítico es el razonamiento, el cual está vinculado al proceso de reflexión, que se conceptualiza como un proceso cognitivo de alto orden, que permite la revisión de información procesada y un cuestionamiento profundo de dicha información (Saadé et al., 2012; Sierra, Carpintero, \& Pérez, 2010). De este modo, la reflexión tendría una función de evaluación profun$\mathrm{da}$, permitiendo evitar la ingenuidad que conlleva el mero proceso de la memorización y asimilación (Melsert \& Bicalho, 2012).

Un segundo elemento es la toma de decisiones, que implica el uso de procesos cognitivos de análisis de la información como pensamiento inductivo y deductivo, identificación de razones y valoración de argumentos (Halpern, 1998; Yang, 2012); procesos que se enfocan en generar el análisis de los datos y razones que permiten decidir respecto a la información. Finalmente, se señala como tercer elemento la resolución de problemas, habilidad que se encuentra relacionada con la anterior en términos de la evaluación de argumentos, pero focalizada en la decisión posterior (Antequera, 2011; Olivares \& Heredia, 2012). Así, la resolución de problemas orientaría el proceso de análisis y revisión de información 
hacia un proceso de juicio evaluativo sistemático que permitiría a los estudiantes buscar las mejores soluciones (Tiwari et al., 2006).

Por otro lado, algunos autores señalan la presencia de un componente no cognitivo en el pensamiento crítico que sería la disposición o motivación (Facione, Facione, \& Giancarlo, 2000; Saiz \& Rivas, 2008; Valenzuela \& Nieto, 2008). Si bien hay diferencias entre ambos conceptos (Valenzuela, Nieto \& Muñoz, 2014), éstas serían de carácter conceptual, puesto que ambos elementos se orientan a la generación de interés y actitudes hacia un objeto de la realidad.

Si bien la disposición es definida como un aspecto más particular que la motivación, el consenso, al parecer, es señalarlo como una disposición ya que de esta manera se ha trabajado en las investigaciones (Facione, 2007; Facione et al., 2000; Valenzuela \& Nieto, 2008). Este componente sería fundamental para el logro de esta habilidad, pues se plantea que aun cuando se disponga de las funciones cognitivas señaladas, si la persona no desea o juzga que no es conveniente aplicar el pensamiento crítico, este no se manifiesta en forma adecuada (Saiz \& Rivas, 2008; Saiz, Rivas, \& Olivares, 2015).

Como conclusión se puede señalar que el pensamiento crítico es una habilidad cognitiva de alto nivel, que permite a la persona disponerse a analizar la información del medio, inferir su validez y propósitos, cuestionar verdades establecidas, reflexionar sobre los propios procesos de pensamiento, y tomar decisiones en base a lo anterior en vez de adoptar un discurso común o una decisión reactiva.

\section{Evaluación del Pensamiento crítico}

Para la evaluación del pensamiento crítico se han construido instrumentos tanto cuantitativos como cualitativos, entre los cuales se pueden indicar la observación directa, los cuestionarios, las discusiones y los portafolios (Calle Álvarez, 2013). Cada modalidad de evaluación incide directamente en la cantidad de individuos posibles de evaluar de forma simultánea, ya que para algunos autores es posible evaluar el pensamiento crítico con instrumentos que se pueden aplicar a un numeroso grupo de personas, como los cuestionarios de respuesta cerrada (Facione, 1990); otros en cambio consideran que lo ideal es trabajar con grupos pequeños y analizar todos los comportamientos de manera cualitativa, como el caso de los instrumentos de respuesta abierta (Marzano \& Costa, 1988).

Cada uno de estos instrumentos está relacionado con modelos y programas específicos que no siempre han informado sobre las propiedades psicométricas que poseen; instituciones como American Colleges and Universities Association (en Butler, 2012) debaten actualmente acerca de la fiabilidad y validez de dichos instrumentos, pues resaltan algunos problemas tanto conceptuales como metodológicos sobre el concepto (Rivas \& Saiz, 2012).

Por un lado, a nivel conceptual, se señala la gran diversidad de conceptualizaciones del pensamiento crítico; y por otro, a nivel metodológico, se plantea el escaso análisis respecto al tipo de formato utilizado para evaluarlo, ya que la mayoría de las pruebas son instrumentos con un formato de respuesta cerrada, por lo tanto, se centran habitualmente en razonamientos simples deductivos, e impiden la exploración de los mecanismos fundamentales implicados en la tarea de pensar críticamente como la argumentación o la resolución de problemas (López, 2012; Olivares \& Heredia, 2012).

Las pruebas existentes se han dividido tradicionalmente en dos grupos, los que utilizan ítems de selección múltiple, con respuestas cerradas; y los que evalúan en base al desarrollo de respuestas abiertas o un ensayo. Los primeros presentan en general buenas cualidades estadísticas (validez y confiabilidad), pero solamente evalúan aspectos predeterminados del pensamiento crítico y presentan dificultades para su repetición. Los de ensayo son más adaptables a las necesidades específicas de cada caso o definición, son más fáciles de repetir, pero su validez de constructo y confiabilidad es limitada (Madariaga \& Schaferchnit, 2013).

Sin embargo, hay algunos intentos de generar propuestas híbridas que recogen ambos tipos de preguntas, como el Test de habilidades de pensamiento crítico de Halpern, el cual presenta 25 situaciones problema, en la que se realiza una pregunta de respuesta cerrada, y luego una de respuesta abierta, de manera que se pueda recoger el procesamiento de la persona por ambas vías de razonamiento (Halpern, 1998; Marin \& Halpern, 2011). Asimismo, la República de Paraguay el año 2007 realizó un test de pensamiento crítico como parte de una política ministerial de mejoramiento de la educación Media (Secundaria), que consistía en una parte de preguntas de respuestas cerradas, y una segunda de análisis de escenas donde se explica argumentativamente la situación (LaFuente, 2009).

De este modo, si bien han existido algunas sistematizaciones (Butler, 2012; Olivares \& Heredia, 2012), estas han sido descriptivas y no analíticas, por lo que no se ha logrado determinar cuáles aspectos de medición del fenómeno están siendo mayormente valorados y cuáles están siendo menos 
valorados. Por otra parte, no se ha profundizado en el análisis de las dimensiones que se han utilizado para medir el pensamiento crítico, lo que ha permitido una proliferación de instrumentos que no permiten determinar si se está evaluando procesos similares o diferentes.

Por lo anterior, el objetivo de la investigación fue analizar estudios sobre medición de pensamiento crítico, identificando los instrumentos de medición más utilizados en la investigación de este tema, y generar un análisis de tópicos relevantes que permitan determinar elementos que se encuentren aun poco estudiados, de manera que se pueda profundizar en ellos.

\section{Materiales y Método}

En este trabajo se utilizó una estrategia de investigación documental. El análisis de los antecedentes sobre medición del pensamiento crítico, se basó en la búsqueda bibliográfica sistemática de publicaciones en las bases de datos Scopus, Scielo, Latindex, Google scholar y Web of Science (WOS).

La búsqueda se centró en una primera fase, en descriptores generales como pensamiento crítico y medición, abarcando todo tipo de publicaciones; se refinó posteriormente la búsqueda con criterios como año de publicación e idioma. La última fase se centró en descartar aquellas investigaciones que consideraban procesos similares, pero de diferente contexto (lectura crítica, teoría crítica); así como de artículos que eran una repetición del mismo estudio con menos variables, o el mismo estudio en diferente idioma.

Se utilizó además, la estrategia de análisis de contenido para examinar las definiciones del concepto, y características de los instrumentos, centrándose en tópicos como dimensiones utilizadas, tipo de respuestas del instrumento, grupo etario evaluado, y, niveles de confiabilidad.

\section{Resultados}

Se encontraron en la primera búsqueda 97 estudios, abarcando desde el año 1980 hasta el año 2016, pero como se observa en la figura 1, con un mayor desarrollo en los años 2011 a 2016; 13 de los 97 estaban en español (13,4\%), uno en portugués $(1 \%)$ y 83 en inglés $(85,6 \%)$. En una segunda fase, se filtró la búsqueda considerando sólo los artículos empíricos, y que mostraran datos de aplicación y validación de los instrumentos, lo que originó un total de 31 estudios (ver figura 1).

En la tercera fase, se seleccionaron ocho estudios de los 31, para realizar un análisis más profundo, dejando de lado aquellos estudios que repetían el mismo instrumento, no señalaban la muestra utilizada o trabajan en una muestra similar a otros. Estos ocho estudios señalaban los instrumentos usado para medir el pensamiento crítico, considerándose en el análisis los autores y el año de publicación del instrumento, las dimensiones que mide, la muestra utilizada en la validación y los niveles de confiabilidad, la cantidad de ítems, y finalmente, el país en que aplicó la validación.

En la revisión de estos últimos estudios, se encontraron los siguientes instrumentos:

1.- Watson-Glaser Critical Thinking (WGCTA; Watson \& Glaser, 1980). Este instrumento se utilizó por primera vez en 1930 con estudiantes y adultos, tiene dos formas de aplicación (con o sin límite de tiempo).La versión original presenta 80 preguntas de opción múltiple, cerradas y contiene cinco sub-escalas: a) inferencia: mide la habilidad de discriminar para encontrar la verdad; b) reconocimiento de supuestos: a través de la cual se mide la capacidad de establecer afirmaciones o negaciones de un proceso; c) deducción: variable que determina la habilidad para razonar deductivamente; $d$ ) interpretación, mide la habilidad para determinar si las generalizaciones son válidas y e) evaluación de argumentos, que discrimina entre argumentos fuertes y débiles. La versión original registra una confiabilidad de .73-.83. También es posible encontrar una versión reducida de 40 preguntas, que en un estudio realizado hace algunos años ha demostrado su validez y confiabilidad, encontrando una confiabilidad medida con alfa de Cronbach de .82 (De Mangione \& De Anglat, 2007; Watson, \& Glaser, 1980).

2.- California Critical Thinking Skills (CCTST; Facione, 1990). Este instrumento fue creado para el contexto universitario de pregrado o postgrado y personas adultas a nivel de ejecutivos. El instrumento permite evaluar cinco habilidades cognitivas; interpretación, análisis, evaluación, explicación e inferencia. El CCTST reporta seis puntuaciones, una global de las habilidades cognitivas del pensamiento crítico y cinco sub-puntuaciones: a) análisis, mide las habilidades de categorización, codificación de significado y clarificación, así como la forma de examinar ideas y detectar y analizar los componentes de un argumento; b) evaluación, se enfoca a las habilidades para calificar y presentar los argumentos y contra-argumentos, establecer resultados y justificar procedimientos; c) inferencia, evalúa la forma de buscar evidencia, hacer conjeturas sobre las alternativas y establecer conclusiones; d) razonamiento 


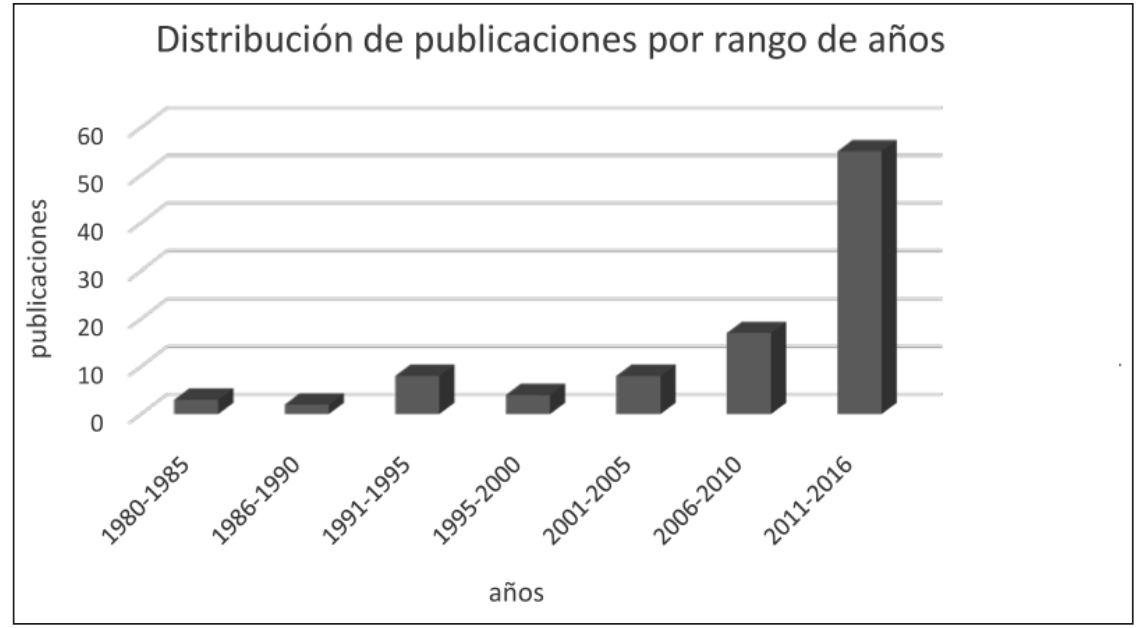

Figura 1. Gráfico de distribución de publicaciones

deductivo, veracidad comprobable de las premisas; e) razonamiento inductivo, asumir los precedentes. La confiabilidad informada de este instrumento es de .78 a .80 (Facione, 1990).

3.- California Critical Thinking Disposition Inventory (CCTDI; Facione et al., 2000). El instrumento surge bajo los auspicios de la Asociación Norteamericana de Filosofía, que se completó y se publicó bajo el título Critical Thinking: A Statement of Expert Consensus for Purposes of Educational Assessement and Instruction (American Philosophical Association), con la coordinación de un grupo multidisciplinario a cargo de Peter Facione (1990). El objetivo del trabajo fue buscar un consenso con respecto a las destrezas intelectuales, así como a las disposiciones personales necesarias para pensar críticamente. El instrumento contiene 75 ítems de respuesta cerrada y posee buenos indicadores de confiabilidad (Facione et al., 2000). El CCTDI se ha desarrollado para población adulta, pero se pueden incluir estudiantes de educación superior y media, tanto profesional como técnica. Para contestar el sujeto evaluado debe señalar el grado de acuerdo o desacuerdo con oraciones que expresan opiniones, valores, creencias y percepciones relacionadas con la reflexión, el razonamiento de juicios y la toma de decisiones. Se reportan indicadores de confiabilidad buenos para el instrumento en general, con un alfa de Cronbach de .90 , y con indicadores de las escalas que van de .72 a .80 (Ruud, Baker, \& Hoover, 2000). Las habilidades intelectuales necesarias, identificadas por consenso, son: Análisis, Inferencia, Interpretación, Explicación, Evaluación y Auto-regulación.

4.- Test de Cornell Critical Thinking (CCTT; Ennis \& Millman, 1985). Este instrumento se planteó con dos niveles $\mathrm{X}$ y Z, siendo dirigido a niños y jóvenes de 9 y 18 años de edad, su estructura es de opción múltiple, consta de 76 elementos que evalúan las habilidades de: 1) inducción, 2) credibilidad de una fuente, 3) observación, 4) semántica, 5) deducción, y 6) identificación de hipótesis. El nivel $\mathrm{X}$ corresponde a una prueba de comprensión, en la que el estudiante selecciona una opción de tres respuestas posibles por cada pregunta. El Nivel Z consta de 52 ítems con tres alternativas de respuesta, evalúa las siguientes habilidades: inducción, credibilidad de una fuente, semántica, predicción y experimentación, falacias, deducción, definición e identificación de hipótesis. Este nivel es para estudiantes universitarios y adultos, aunque también puede ser aplicada en estudiantes avanzados de educación básica o media. Gordón (1994) realizó un estudio con el propósito de aportar con una traducción al español válida y confiable del nivel $\mathrm{Z}$ del instrumento, a la que denominó Prueba Cornell para Pensamiento Crítico. Su propósito fue determinar el efecto en la adquisición de destrezas de pensamiento crítico al enseñar a estudiantes de un curso introductorio de enfermería algunas destrezas de pensamiento crítico (Calle Álvarez, 2013).

5.- Halpern Critical Thinking Assessmentusing Everyday Situations (HCTAES; Halpern, 1998), el que a través de 25 preguntas de respuestas cerradas y abiertas (50 en total), busca medir de manera más amplia el logro de la habilidad, acompañándolo con una medición más objetiva y certera. El HCTAES considera cinco dimensiones: testeo de hipótesis, razonamiento verbal, argumentación, probabilidades e incertidumbre, y finalmente, resolución de problemas (Marin \& Halpern, 2011). Este instrumento mostró buenos indicadores de confiabilidad con un alfa de Cronbach de .88-.77, en muestras de diversos países (Halpern, 1998). 
6.- Pensamiento crítico Salamanca (PENCRISAL; Rivas \& Saiz, 2012; Saiz \& Rivas, 2008).El cuestionario cuenta con 35 ítems que se configuran en 5 factores: razonamiento deductivo, inductivo y práctico, toma de decisiones, y solución de problemas, a razón de 7 ítems por factor. Los ítems se presentan en un formato informatizado a fin de minimizar el cansancio y obtener resultados de manera más rápida y eficiente. En la evaluación del instrumento se utiliza un sistema de escalamiento cuantitativo, cuyo rango de valores se sitúa entre 0 y 70 puntos para la puntuación global y entre 0 y 14 para cada una de las cinco escalas. La consistencia interna de los 35 ítems se estimó en Alfa de Cronbach, obteniendo un valor global de 63 .

7.- Tareas de Pensamiento Crítico (TPC; Miranda, 2003). En el contexto de una investigación chilena en la queparticiparon352 docentes de educación básica de un programa de pensamiento crítico, se utilizó una versión adaptada para Chile, cuyos resultados mostraron buenas propiedades psicométricas. Este instrumento fue utilizado nuevamente (Miranda, Zambrano, \& Jelvez, 2010) en un estudio con estudiantes de Pedagogía en Castellano. Estudios en que también se indican buenos niveles de confiabilidad con un alfa de Cronbach de .87 .

8.- Test de Pensamiento Crítico (TPC) de Paraguay. Este instrumento fue desarrollado por el Sistema Nacional de Evaluación del Proceso educativo (SNEPE) del Ministerio de Educación y Cultura de Paraguay (2007). Consiste en dos tareas, una de las cuales tenía una hoja de ejercicios para responder a situaciones, que consideraba 3 dimensiones y cinco sub habilidades; y una segunda parte, de respuesta abierta a partir de imágenes que debían ser explicadas (LaFuente, 2009; MEC, 2007); presenta buena confiabilidad (alfa de .70 y .71). La tabla 1, muestra una síntesis de la información arriba señalada, incluyendo además la muestra, país de validación y cantidad de ítems.

\section{Discusión}

Como se ha mencionado anteriormente, el pensamiento crítico es un constructo muy complejo, definido desde marcos teóricos muy diversos, como resultado de instrumentos de diferente naturaleza (Rivas \& Saiz, 2012).

La revisión documental muestra que en los países de Latinoamérica existe una escasez de experiencias que permitan fomentar de manera didáctica dichas habilidades en la formación universitaria, por lo que los estudiantes estarían ingresando con pocas herramientas de pensamiento crítico a la educación superior (Madariaga \& Schaffernicht, 2013). Aun cuando existen experiencias e instrumentos desarrollados en Chile y Paraguay, son escasas experiencias en comparación con las desarrolladas en América del Norte, Europa y el Oriente.

A partir del análisis de los instrumentos de medición del Pensamiento crítico, se discuten algunas ideas en relación con las dimensiones señaladas, tipo de respuestas, grupo etario al que se dirige, y niveles de confiabilidad reportados.

a.- Análisis de las dimensiones. Los instrumentos analizados presentan entre 3 a 7 dimensiones, variando en cada instrumento según el constructo teórico del autor. Por ejemplo, Ennis y Millman (1985), valida un instrumento con 7 dimensiones, hacia los años 90, Facione plantea 5 dimensiones, mientras el mismo autor más tarde (Facione, 1990), reconsidera, 7 habilidades, construidas bajo otro modelo. El test de Halpern (Halpern, 1998, 2006), incorpora habilidades como hipótesis, y probabilidad e incertidumbre. En tanto, el test PENCRISAL (Saiz \& Rivas, 2008) establece 5 habilidades, pensando en el Razonamiento práctico y la Resolución de problemas. Finalmente, el test de Tareas de Pensamiento crítico (TPC) de Miranda (2003), validado en Chile, establece, tres habilidades como: Indagación, análisis y comunicación. El test TPC, es un instrumento de desempeño, adaptación del Task of Critical Thinking (TCT), basado en preguntas de ensayo, contextualizado en un formato de informaciones científicas relacionadas al fenómeno del Niño Oscilación Sur (ENOS). Presenta una validación en un grupo de profesores chilenos (Miranda, 2003) y en estudiantes de pedagogía del área de lenguaje (Miranda et al., 2010).

b.- Tipo de respuestas. La mayoría de las pruebas que evalúan pensamiento crítico son instrumentos con formato de respuesta cerrada, que impiden la exploración de los mecanismos fundamentales del pensamiento implicados en la tarea de responder a un test (Rivas \& Saiz, 2012). A juicio de estos autores, existen al menos tres problemas graves en la mayoría de las pruebas estandarizadas: el primero, que no se sabe bien lo que miden, el segundo, que no activan las habilidades fundamentales objeto de la medida y finalmente, que los problemas son artificiales y alejados de la actividad cotidiana de la gente. Por ello, el test HCTAES (Halpern Critical Thinking Assessment Using Everyday Situations; Halpern, 1998), permite resolver esta dificultad. Este instrumento se centra en los procesos de pensamiento y los ítems que se proponen en la prueba son situaciones 
Tabla 1

Instrumentos para medir pensamiento crítico (elaboración propia)

\begin{tabular}{|c|c|c|c|c|c|c|c|c|}
\hline Instrumento & Autor & Año & Dimensiones & $\begin{array}{l}\text { Tamaño } \\
\text { Muestra }\end{array}$ & $\begin{array}{l}\text { Confiabilidad } \\
\text { v estadístico }\end{array}$ & $\begin{array}{l}\text { Tipo de } \\
\text { respuesta }\end{array}$ & $\begin{array}{c}\text { País de } \\
\text { validación }\end{array}$ & $\begin{array}{l}N^{\circ} \text { de } \\
\text { ítem }\end{array}$ \\
\hline $\begin{array}{l}\text { WGCTA } \\
\text { (Watson- } \\
\text { Glaser Critical } \\
\text { Thinking } \\
\text { Assessment) }\end{array}$ & $\begin{array}{l}\text { Watson y } \\
\text { Glaser }\end{array}$ & 1980 & $\begin{array}{ll}\text { 1. } & \text { Inferencia } \\
\text { 2. } & \text { Reconocimiento de } \\
& \text { supuestos } \\
\text { 3. } & \text { Deducción } \\
\text { 4. } & \text { Interpretación } \\
\text { 5. } & \text { Evaluación de } \\
& \text { argumentos } \\
\end{array}$ & 102 & $\begin{array}{l}0,82 \text { Alfa de } \\
\text { Cronbach }\end{array}$ & Cerrada & USA & 80 \\
\hline $\begin{array}{l}\text { CCTST } \\
\text { (California } \\
\text { Critical } \\
\text { Thinking } \\
\text { Skills Test) }\end{array}$ & Facione & 1990 & $\begin{array}{ll}\text { 1. } & \text { Interpretación } \\
\text { 2. } & \text { Inferencias } \\
\text { 3. } & \text { Análisis } \\
\text { 4. } & \text { Evaluación } \\
\text { 5. } & \text { Explicación }\end{array}$ & 133 & $\begin{array}{l}0.78 \text { a } 0.80 \\
\text { mitades } \\
\text { partidas de } \\
\text { Spearman }\end{array}$ & Cerrada & USA & 34 \\
\hline $\begin{array}{l}\text { CCTDI } \\
\text { (California } \\
\text { Critical } \\
\text { Thinking } \\
\text { disposition } \\
\text { inventory) }\end{array}$ & Facione & 2001 & $\begin{array}{ll}\text { 1. } & \text { Credibilidad } \\
\text { 2. } & \text { Mentalidad abierta } \\
\text { 3. } & \text { Capacidad Análisis } \\
\text { 4. } & \text { Sistematicidad } \\
\text { 5. } & \text { Confianza en } \\
& \text { Razonamiento } \\
\text { 6. } & \text { Curiosidad } \\
\text { 7. } & \text { Madurez de Juicio } \\
\end{array}$ & 193 & $\begin{array}{l}0.90 \text { alfa de } \\
\text { Cronbach }\end{array}$ & Cerrada & USA & 75 \\
\hline $\begin{array}{l}\text { CCTT } \\
\text { (Cornell } \\
\text { Critical } \\
\text { Thinking Test) }\end{array}$ & $\begin{array}{l}\text { Ennis y } \\
\text { Millman, }\end{array}$ & 1985 & $\begin{array}{ll}\text { 1. } & \text { Argumentación } \\
\text { 2. } & \text { Inducción } \\
\text { 3. } & \text { Deducción } \\
\text { 4. } & \text { Credibilidad } \\
\text { 5. } & \text { Semántica } \\
\text { 6. } & \text { Definiciones } \\
\text { 7. } & \text { Predicción } \\
\end{array}$ & S.I ${ }^{1}$ & $\begin{array}{c}0.62-0.64 \\
\mathrm{Kr}-20\end{array}$ & Cerrada & S.I. & 62 \\
\hline $\begin{array}{l}\text { HCTAES } \\
\text { (Halpern } \\
\text { Critical } \\
\text { Thinking } \\
\text { Assessment } \\
\text { using } \\
\text { Everyday } \\
\text { Situations) } \\
\end{array}$ & Halpern & 2006 & $\begin{array}{ll}\text { 1. } & \text { Razonamiento Verbal } \\
\text { 2. Análisis de argumentos } \\
\text { 3. Prueba de hipótesis } \\
\text { 4. } \\
\text { Probabilidad e } \\
\text { incertidumbre } \\
\text { 5. Toma de decisiones/ } \\
\text { resolución de } \\
\text { problemas }\end{array}$ & $60^{2}$ & $\begin{array}{l}0.88-0.77 \\
\text { Alfa de } \\
\text { Cronbach }\end{array}$ & $\begin{array}{l}\text { Mixta: } \\
\text { Abiertas y } \\
\text { cerradas }\end{array}$ & Colombia & 50 \\
\hline $\begin{array}{l}\text { PENCRISAL } \\
\text { (Pensamiento } \\
\text { crítico } \\
\text { Salamanca) }\end{array}$ & $\begin{array}{l}\text { Saiz y } \\
\text { Rivas }\end{array}$ & 2012 & $\begin{array}{ll}\text { 1. } & \text { Razonamiento } \\
\text { 2. } & \begin{array}{l}\text { Inductivo } \\
\text { Razonamiento }\end{array} \\
\text { 3. } & \text { Deductivo } \\
\text { 4. } & \text { Razonamiento } \\
\text { 5. } & \text { Toma de decisiones/ } \\
\text { 6. } & \begin{array}{l}\text { Resolución de } \\
\text { problemas }\end{array} \\
\end{array}$ & 715 & $\begin{array}{c}0.73 \\
\text { Alfa de } \\
\text { Cronbach }\end{array}$ & Abierta & España & 35 \\
\hline $\begin{array}{l}\text { Tareas de } \\
\text { pensamiento } \\
\text { crítico (TPC) }\end{array}$ & Miranda & 2003 & $\begin{array}{ll}\text { 1. } & \text { Indagación } \\
\text { 2. } & \text { Análisis } \\
\text { 3. } & \text { Comunicación } \\
\end{array}$ & 352 & $\begin{array}{c}0.87 \\
\text { Alfa de } \\
\text { Cronbach }\end{array}$ & Abierta & Chile & 14 \\
\hline $\begin{array}{l}\text { Test de } \\
\text { Pensamiento } \\
\text { Crítico (MEC } \\
\text { Paraguay) }\end{array}$ & $\begin{array}{c}\text { Servicio } \\
\text { Nacional de } \\
\text { Evaluación } \\
\text { del proceso } \\
\text { educativo }\end{array}$ & 2006 & $\begin{array}{ll}\text { 1. } & \text { Silogismos } \\
\text { 2. } & \begin{array}{l}\text { Proposiciones condi- } \\
\text { cionales }\end{array} \\
\text { 3. } & \begin{array}{l}\text { Identificación de } \\
\text { supuestos }\end{array} \\
\text { 4. } & \begin{array}{l}\text { Diferenciación entre } \\
\text { hechos y opiniones }\end{array} \\
\text { 5. } & \begin{array}{l}\text { Inferencias a partir de } \\
\text { datos }\end{array} \\
\text { 6. } & \begin{array}{l}\text { Interpretaciones de } \\
\text { situaciones, escenas, } \\
\text { lúdicas o conflictivas }\end{array}\end{array}$ & $2.816^{2}$ & $\begin{array}{c}0.70 \text { primer } \\
\text { componente } \\
0,71 \text { segundo } \\
\text { componente } \\
\text { Alfa de Cronbach }\end{array}$ & $\begin{array}{l}\text { Mixta } \\
\text { (Abiertas y } \\
\text { cerradas) }\end{array}$ & Paraguay & 6 \\
\hline
\end{tabular}

${ }^{1} \mathrm{SI}=\mathrm{Sin}$ Información. ${ }^{2}$ Estudiantes de secundaria (15-18 años) 
que describen problemas cotidianos que se deben resolver mediante respuestas abiertas y cerradas.

Más tarde, en el intento de mejorar la versión de HCTAES, surge la prueba PENCRISAL de respuesta abierta, cuyas características son: 1) la utilización de ítems que sean situaciones cotidianas, 2) el uso de diferentes dominios, con la intención de valorar el grado de generalización de las habilidades, 3 ) un formato de respuesta abierta, que posibilita la exploración de los procesos de pensamiento, y 4) el empleo de situacionesproblema de respuesta única que permite evaluar el mecanismo de pensamiento correspondiente y facilita la cuantificación de los ítems (Rivas \& Saiz, 2012; Saiz \& Rivas, 2008). Sin embargo, la limitante de esta prueba es el sistema de corrección que requiere de evaluadores expertos y el tiempo de los protocolos de respuesta es elevado.

c.- Grupo etario. El primer instrumento WGCTA, fue aplicado tanto a estudiantes como adultos. Luego, CCTST, aplicado a estudiantes de pre y postgrado, mientras CCTT, dirigido a estudiantes de 9 y 18 años. A pesar de la variada existencia de instrumentos, la mayoría han sido utilizados en estudiantes universitarios y adultos (Pencrisal, HCTAES, TPC, CCTT, CTDI, CCTST) y unos pocos a estudiantes de secundaria, como el HCTAES y el del Ministerio de Educación del Paraguay. Sin embargo, hasta ahora no se ha discriminado en relación al tipo de preguntas por edad o nivel educativo.

d.- Nivel de confiabilidad. Las diferentes iniciativas de evaluación hasta ahora desarrolladas, la mayoría, han mostrado problemas graves de validez, que han puesto en tela de juicio su viabilidad, siendo los test de respuesta cerrada los que presentan los más bajos niveles de confiabilidad. Por su parte, Saiz y Rivas (2008), resuelven los problemas de validez, producción y complejidad en la prueba PENCRISAL, analizando qué es lo que consigue medir para ello asumen el modelo de medida del HCTAES, pero corrigiendo sus limitaciones e incorporando una propuesta nueva que resuelve el problema de la validez.

El análisis de estas diferentes tipologías, nos permite agrupar las habilidades citadas en tres grandes dimensiones. La primera concierne a las habilidades vinculadas a la capacidad de depurar las informaciones a través de hacer preguntas, concebir y juzgar definiciones, distinguir los diferentes elementos de una argumentación, de un problema, de una situación o de una tarea, identificar y aclarar los problemas importantes. La segunda dimensión abarca las habilidades vinculadas a la capacidad de elaborar un juicio sobre la fiabilidad de las informaciones (juzgar la credibilidad de una fuente de información, juzgar la credibilidad de una información, identificar los presupuestos implícitos, juzgar la validez lógica de una argumentación). En cuanto a la tercera, se refiere a las habilidades relacionadas con la capacidad de evaluar las informaciones -obtener conclusiones apropiadas, realizar generalizaciones, inferir, formular hipótesis, generar y reformular de manera personal una argumentación, un problema, una situación o una tarea- (Piette, 1998).

A través de los diferentes instrumentos aquí analizados, se puede visualizar que las habilidades de argumentación también han sido relevadas por diversos autores quienes señalan que una forma argumentada de entender el mundo, ocupa un lugar central en la actividad científica (Pinochet, 2015). Finalmente, la argumentación y consecuentemente el pensamiento crítico son habilidades coherentes con las demandas del nuevo siglo, ya que la cantidad de informaciones y datos que nos llegan cotidianamente, y frente a la que debemos tomar decisiones, es muy grande. Asimismo, las demandas del sistema educativo a nivel de aula, extensible a todas las disciplinas escolares exigen a los estudiantes que argumenten sus respuestas, por lo que resulta indispensable mejorar la base argumentativa en ellos (Campillo \& Guerrero, 2013).

\section{Conclusiones}

A nuestro juicio, el desarrollo de habilidades de pensamiento crítico debiera ser una prioridad a ser instalada en la educación media y superior, especialmente en la formación universitaria, pues permitiría el cambio en las prácticas pedagógicas y la calidad de la educación (LaFuente, 2009; Marin \& Halpern, 2011). Al respecto, Saiz y Rivas (2008) plantean que estas habilidades se basan en argumentar, decidir y resolver problemas, de manera que la persona pueda pensar mejor y generar cambios en su vida, siendo básicas para el razonamiento científico y crítico. La idea es poner en práctica una serie de habilidades cognitivas y procedimentales que permitan construir, comunicar y evaluar el conocimiento científico mediante la argumentación (Pinochet, 2015).

Si bien el factor cognitivo es el que predomina mayormente, y da además la imagen de que el pensamiento crítico es un modo de usar el pensamiento de forma eficaz, la incorporación de la motivación como factor potenciador del mismo, es relevante para entender la complejidad del mismo, ya que 
no solo debe ser considerado como un proceso cognitivo, sino también disposicional.

En este sentido la motivación genera el interés necesario para mantener activados los procesos cognitivos que se desarrollan y orientan el metaconocimiento crítico (Rivas \& Saiz, 2012; Saiz, Rivas, \& Olivares, 2015). Del mismo modo Daniel y Auriac (2012), rescatan la importancia del componente disposicional señalando que el pensamiento crítico no está solo conformado por complejas habilidades cognitivas, sino que además por un espíritu crítico, el cual estaría relacionado tanto con habilidades dialógicas del sujeto, como con la disposición a utilizarlo.

Aun cuando quizás sea adecuado contar con diferentes maneras de entender el pensamiento crítico, y así mismo de poder medirlo, es necesario tener una base común para lograr compartir experiencias y evidencias empíricas. Esta revisión da cuenta de lo difícil que es esto, y además, de lo lejos que nos encontramos ya que lo que ha predominado es la ultraespecialización de los instrumentos para abracar habilidades mucho más focalizadas.

Surge la idea de visibilizar la habilidad de pensamiento crítico de una manera más integrada. Es decir, considerar las habilidades cognitivas y metacognitivas como base y por otro lado la posibilidad de generar autorregulación y motivación a fin de lograr una disposición crítica, que implica estar abierto a enfoques múltiples para tomar decisiones e intervenir en la realidad social. Estos componentes definirían el pensamiento crítico como un proceso sofisticado y complejo, que incluiría habilidades cognitivas, de metacognición, disposición al juicio crítico, y colaboración frente a resolución de problemas (Olivares \& Heredia, 2012; Rivas \& Saiz, 2012; Tiwari et al., 2006; Yang, 2012).

Dicha perspectiva integral es a nuestro juicio, una manera más adecuada de entender este complejo constructo, pero a la vez, necesario para el mejoramiento de los programas de promoción que buscan fortalecer las habilidades en las personas, permitiendo una base común.

\section{Referencias}

Almeida, L., \& Rodríguez, A. (2011).Critical thinking: Its relevance for education in a Shifting society, Revista de Psicología, 29(1), 175-195. Recuperado de: http://www. scielo.org.pe/scielo.php?script=sci_arttext\&pid=S0254$92472011000100007 \& \operatorname{lng}=$ es\&nrm $=$ iso

Antequera, G. (2011). La promoción del pensamiento crítico en el aprendizaje basado en problemas (ABP). Un análisis a partir de los instrumentos de medición. Observar, 5, 68-94. Recuperado de: http://www.raco.cat/ index.php/Observar/article/viewFile/247662/331621
Bailin, S., Case, R., Coombs, J., \& Daniels, L. (1999). Common misconceptions of critical thinking. Journal of curriculum studies, 31, 269-283. Recuperado de: http://www.iskconeducation.org/download/ BAILIN,CASE,COOMBS\%20\&\%20DANIELS\%20 - $\% 20$ Common $\% 20$ misconceptions $\% 20$ of $\% 20$ critical\%20thinking.pdf

Black, B. (2012). An overview of a programme of research to support the assessment of critical thinking. Thinking Skills and Creativity, 7, 122-133. doi:10.1016/j. tsc. 2012.04 .003

Butler, H. A. (2012). Halpern Critical Thinking Assessment predicts real-world outcomes of critical thinking. Applied Cognitive Psychology, 26(5), 721-729. doi:10.1002/acp.2851

Cassany, D. (2005). Los significados de la comprensión crítica. Revista lectura y vida, 26(3), 32-45. Recuperado de: http://www.lecturayvida.fahce.unlp.edu.ar/numeros/ a26n3/26 03 Cassany.pdf

Campillo, P., \& Guerrero, J. A. (2013). El ABP y el diagrama Heurístico como herramientas para desarrollar la argumentación escolar en las asignaturas de ciencias. México: UNAM. doi:10.1590/s151673132013000300002

Calle Alvarez, G. (2013). Construcción de argumentos durante la producción de textos digitales Revista Científica Guillermo de Ockham, 11(2), 101-114. Recuperado de: http://www.redalyc.org/pdf/1053/105329737008.pdf

Daniel, M., \& Auriac, E. (2011).Philosophy, Critical Thinking and Philosophy for Children.Educational Philosophy and Theory, 43(5),415-435. doi:10.1111/j.14695812.2008.00483.x

Davies, M. (2013). Critical thinking and the disciplines reconsidered. Higher Education Research \& Development, 32(4), 529 - 544. doi:10.1080/072943 60.2012.697878

De Mangione, E, \& De Anglat, H.(2007) Evaluación de la competencia crítica a través del test de Watson-Glaser. Exploración de sus cualidades psicométricas. Revista de Psicología, 3(6), 1-14. Recuperado de: http:// bibliotecadigital.uca.edu.ar/repositorio/revistas/revistapsicologia06.pdf

Ennis, R. H., \& Millman, J. (1985). Cornell critical thinking test, level X. Pacific Grove, CA: Midwest Publications.

Facione, P.(1990). Critical thinking: A statement of expert consensus for purposes of educational assessment and instruction. Millbrea, CA: The California Academic Press.

Facione, P. (2007). Pensamiento crítico. ¿Qué es y por qué es importante? Actualización. Documento online, 1-22. Recuperado de: http://www.eduteka.org/ PensamientoCriticoFacione.php

Facione, P. A., Facione, N. C., \& Giancarlo, C. A. F. (2000). The disposition toward critical thinking: Its character, measurement, and relationship to critical thinking skill. Informal Logic, 20(1), 61-84. Recuperado de: https://www.insightassessment.com/About-Us/ Measured-Reasons/pdf-file/The-Disposition-TowardCritical-Thinking-Its-Character-Measurement-andRelationship-to-Critical-Thinking-Skill-PDF

Gordón, L. (1994). El efecto de enseñar las destrezas del pensamiento crítico en un curso introductorio de enfermería. Rev. Latino-Am. Enfermagem [Internet]. 2(2), 115-127. doi:10.1590/S0104-11691994000200009

Hager, P., \& Kaye, M. (1992).Critical Thinking in Teacher Education: A Process-Oriented Research Agenda. Australian Journal of Teacher Education, 17(2), 26-33. Recuperado de: http://ro.ecu.edu.au/cgi/viewcontent. cgi? article $=1218 \&$ context $=$ ajte

Halpern, D. (1998). Teaching critical thinking for transfer across domains dispositions, skills, structure training, and metacognitive monitoring. American Psychologist, 53(4), 449-455. doi:10.1037/0003-066X.53.4.449 
Halpern, D. (2006). The nature and nurture of critical thinking. In R. J. Sternberg, H. L. Roediger, \& D. F. Halpern (Eds.), Critical thinking in psychology (pp. 1-14). Cambridge, UK: Cambridge UniversityPress.

LaFuente, M. (2009). La Experiencia del Sistema Nacional de Evaluación del Proceso Educativo, SNEPE, en Paraguay. Aprendizajes y desafíos. Revista Iberoamericana de Evaluación Educativa [online], 2(1). Recuperado de: http:// rinace.net/riee/numeros/vol2-num1/art3 htm.html

López, G. (2012). Pensamiento crítico en el aula. Docencia e Investigación, XXXVII(22), 41-60. Recuperado de: http:// educacion.to.uclm.es/pdf/revistaDI/3_22_2012.pdf

Madariaga, P., \& Schaffernicht, M. (2013).Uso de objetos de aprendizaje para el desarrollo del pensamiento crítico. Revista de Ciencias Sociales (RCS), XIX, (3), 472 - 484. Recuperado de: http://www.redalyc.org/ pdf/280/28028572010.pdf

Marin, L., \& Halpern, D. F. (2011). Pedagogy for developing critical thinking in adolescents: Explicit instruction produces greatest gains. Thinking Skills and Creativity. 6(1), 1-13. doi:10.1016/j.tsc.2010.08.002

Marzano, R.J. \& Costa, A. (1988). Question: Do Standardized Tests Measure General Cognitive Skills? Answer: No. Educational Leadership, 45(8), 66-71. Recuperado de: http://ascd.com/ASCD/pdf/journals/ed_lead/ el_198805 marzano.pdf

Melsert, A.L.\&Bicalho, P. P. (2012).Desencontros entre umaprática crítica empsicologia e concepções tradicionais em educação. Revista Semestral da Associação Brasileira de Psicologia Escolar e Educacional, 16(1), 153-160. doi:10.1590/S141385572012000100016

Ministerio de Educación y Cultura. MEC (2007). Informe de Resultados Test de Pensamiento Crítico. Segundo Curso. Sistema Nacional de Evaluación del Proceso Educativo. Programa Reforma Joven. Recuperado de: http://www.oei.es/historico/pdfs/informe-resultadossnepe5.pdf

Miranda, C. (2003). El pensamiento crítico en docentes de EducaciónGeneralBásicaen Chile: un estudiodeimpacto. Estudios Pedagógicos, 29, 39-54. Recuperado de: http://mingaonline.uach.cl/scielo.php?script=sci arttext\&pid=S0718$07052003000100003 \& 1 \mathrm{ng}=\mathrm{es} \& \mathrm{nrm}=$ is o

Miranda, C., Zambrano, F., \& Jélvez, M. (2010). ¿Incide la formación inicial en el desarrollo del pensamiento crítico de los estudiantes de pedagogía? Insumos desde un estudio de caso para un debate en curso. Boletín de Investigación Educacional, 24(1), 79-89.

Olivares, S., \& Heredia, Y. (2012). Desarrollo del pensamiento crítico en ambientes de aprendizaje basado en problemas en estudiantes de educación superior. Revista Mexicana de Investigación Educativa, 17(54), 759-778. Recuperado de: http://www.redalyc.org/ pdf/140/14023127004.pdf

Phan, H. P. (2010). Critical thinking as a self-regulatory process component in teaching and learning.Psicothema, 22(2), 284-292. Recuperado de: http://www.psicothema.com/ PDF/3728.pdf

Paul, R., \& Elder, L. (2003). La mini-guía para el Pensamiento crítico. Conceptos y herramientas. Ed. Fundación para el Pensamiento Crítico. Recuperado de: www. criticalthinking.org

Pinochet, J. (2015). El modelo argumentativo de Toulmin y la educación en ciencias: una revisión argumentada. Ciência \& Educação (Bauru), 21(2), 307-327. doi:10.1590/1516-731320150020004
Piette, J. (1998). Una educación para los medios centrada en el desarrollo del pensamiento crítico. En Alfonso Gutiérrez Martín (coord.). Formación del profesorado en la sociedad de la información. Segovia: EU Magisterio-Universidad de Valladolid. Recuperado de: http://www.quadernsdigitals. net/datos web/biblioteca/1 776/enLinea/5.htm

Rivas, S. F. \&Saiz, C. (2012). Validación y propiedades psicométricas de la prueba de pensamiento crítico PENCRISAL. Revista Electrónica de Metodología Aplicada, 17(1), 18-34. Recuperado de: http://www.pensamientocritico.com/archivos/validapencrisalpub.pdf

Rudd, R., Baker, M., \& Hoover, T. (2000). Undergraduate agriculture student learning styles and critical thinking abilities: Is there a relationship? Journal of Agricultural Education, 41(3), 2-12. doi:10.5032/jae.2000.03002

Saadé, R., Morin, D., \& Thomas, J. (2012).Critical thinking in E-learning environments.Computers in Human Behavior, 28, 1608-1617. doi:10.1016/j. chb.2012.03.025

Saiz, C. \& Rivas, S. (2008). Evaluación en pensamiento crítico: una propuesta para diferenciar formas de pensar. Ergo, Nueva Época, 22-66. Recuperado de: http://www. pensamiento-critico.com/archivos/evaluarpcergodf.pdf

Saiz, C., Rivas, S.F., \& Olivares, S. (2015). Collaborative learning supported by rubrics improves critical thinking. Journal of the Scholarship of Teaching and Learning. 15 (1), 10-19. doi:10.14434/josotl.v15i1.12905

Sánchez, I. (2012). Evaluación de una Renovación Metodológica para un Aprendizaje Significativo de la Física. Formación Universitaria, 5(5), 51-65. doi:10.4067/S0718-50062012000500006

Sierra, J., Carpintero, E. \& Pérez, L. (2010). Pensamiento crítico y capacidad intelectual. Faísca, 15(17), 98 - 110. Recuperado de: https://dialnet.unirioja.es/descarga/ articulo/3548104.pdf

Tiwari, A., Lai, P., So, M., \& Yuen, K. (2006). A comparision of effects of problem based learning and lecturing on the development of students' critical thinking. Medical Education,40(6), 547-554. doi:10.1111/j.13652929.2006.02481.x

Tung, C.A., \& Chang, S.Y. (2009).Developing Critical Thinking through Literature Reading.Feng Chia Journal of Humanities and Social Sciences,19, $287-$

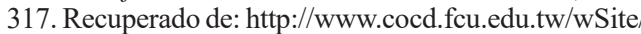
publicfile/Attachment/f1262069682958.pdf

Valenzuela, J., \& Nieto, A. (2008).Motivación y Pensamiento Crítico: Aportes para el estudio de esta relación. Revista electrónica de Motivación y Emoción, 28(1), 1-8. Recuperado de: http://reme.uji.es/articulos/numero28/ article3/article3.pdf

Valenzuela, J., Nieto, A., \& Muñoz, C. (2014). Motivación y disposiciones: enfoques alternativos para explicar el desempeño de habilidades de pensamiento crítico. Revista electrónica de investigación educativa, 16(3), 16-32. Recuperado de:http://www. scielo.org.mx/scielo.php?script $=$ sci arttext\&pid=S1607$40412014000300002 \& \operatorname{lng}=$ es\&tlng $=$ es.

Watson, G., \& Glaser, E. M. (1980).Critical thinking appraisal, forms A and B. New York: Harcourt, Brace and Wold.

Yang, Y. T. (2012).Cultivating critical thinkers: Exploring transfer of learning from pre-service teacher training to classroom practice.Teaching and Teacher Education, 28 1116 -1130. doi:10.1016/j.tate.2012.06.007

Para citar este artículo:

Ossa-Cornejo, C.J., Palma-Luengo, M.R., Lagos-San Martín, N.G., Quintana-Abello, I.M., \& Díaz-Larenas, C.H. (2017). Análisis de instrumentos de medición del pensamiento crítico. Ciencias Psicológicas, 11(1), 19 - 28. doi: 10.22235/cp.v11i2.1343 